\title{
Solerillas de hormigón y plástico reciclado para la conformación de jardineras: hacia un producto más sustentable
}

\author{
Claudia Valderrama-Ulloa, Francisco Parra, Carlos R. Marín-Uribe y Gonzalo Rodríguez-Grau \\ Facultad de Ingeniería, Escuela de Construcción Civil, Pontificia Universidad Católica de Chile, Av. Vicuña Mackenna \\ 4860, Macul, Santiago - Chile (correo-e: c.valderrama@uc.cl, fdparra@uc.cl, crmarin@uc.cl, grodriguezg@uc.cl).
}

Recibido Sep. 8, 2020; Aceptado Nov. 4, 2020; Versión final Dic. 19, 2020, Publicado Abr. 2021

\begin{abstract}
Resumen
El principal objetivo de este estudio es reutilizar el hormigón de desecho de prácticas académicas y plásticos reciclados tipo PET (Polietileno Tereftalato) para la conformación de jardineras. Se propuso un diseño innovador de solerillas con pesos que oscilan entre 15 y $25 \mathrm{~kg}$. Se caracterizan las mezclas de hormigón (0, 0,5 y $1,0 \%$ PET) y se hace un análisis de los aspectos de sustentabilidad de la solución. Los resultados indican que es posible alcanzar la resistencia mínima a la compresión de $15 \mathrm{MPa}$ para todas las mezclas estudiadas. Con esta solución, se reutilizan 6 toneladas de hormigón al año, promoviendo el reciclado de hasta 40 botellas PET por solerilla, evitando anualmente la emisión de $40,66 \mathrm{kgCO}_{2}$ y el consumo energético de 1.345,40 kWh. Se concluye que la solución de elementos prefabricados tipo solerilla para la construcción de jardineras es una alternativa para reducir hormigones en estado fresco que se desechan y para reciclar plástico del tipo PET.
\end{abstract}

Palabras clave: elemento prefabricado; PET; residuos; emisiones de $\mathrm{CO}_{2}$; consumo energético

\section{Concrete and recycled plastic curbs for shaping planters: towards a more sustainable product}

\begin{abstract}
The primary objective of this research study is to reuse waste concrete discarded from academic teachings and to recycle PET (Polyethylene Terephthalate) plastics to make garden planters. An innovative design of concrete curbs is proposed with weights ranging between 15 and $25 \mathrm{~kg}$. Concrete mixtures with different PET additions $(0,0.5$, and $1.0 \%)$ are characterized experimentally and an analysis of sustainability solution aspects is performed. The results show that discarded concrete can achieve the minimum resistance to compression of $15 \mathrm{MPa}$ for all the mixtures studied. Using this solution, it is estimated that six tons of concrete are reused per year, up to 40 PET bottles per concrete curb are recycled, $40.66 \mathrm{~kg}$ of $\mathrm{CO}_{2}$ emissions are avoided annually, and $1,345.40 \mathrm{kWh}$ of energy consumption are saved. It is concluded that the concrete curb mixture developed from recycled materials to make garden planters is an excellent alternative to reduce waste concrete and to recycle PET plastics.
\end{abstract}

Keywords: precast element; recycled plastic; waste; $\mathrm{CO}_{2}$ emissions; energy consumption 


\section{INTRODUCCIÓN}

En los últimos años se ha incrementado el interés para que todos los procesos y procedimientos desarrollados por las empresas e instituciones estén enmarcados dentro del concepto de la sustentabilidad, promoviendo el crecimiento económico pero con un equilibrio en los aspectos social y medioambiental. Las empresas que administran grandes zonas verdes no son la excepción y están siendo desafiadas constantemente por situaciones que requieren soluciones prácticas y eficientes. Un ejemplo de ello es la problemática que presentan los parques, plazas o jardines en lo relativo al mantenimiento de sus áreas verdes y los costos asociados, específicamente a lo relacionado sobre la recolección de las hojas caídas de los árboles. En determinadas épocas del año existe un aumento de la cantidad de hojas caídas por lo que su recolección exige mayor tiempo de trabajo e inversión de recursos económicos. Además, estas hojas colmatan los desagües dificultando la evacuación de las aguas y el correcto mantenimiento de esta infraestructura. El principal interés es que estas hojas se puedan almacenar en sistemas de contención cercanos a las áreas de recolección y distribuidos en todas las zonas verdes, para optimizar los tiempos de traslado del personal de mantenimiento, aumentar su productividad, mejorar los procesos de trabajo y disminuir los costos asociados.

Para dar una solución a este desafío se detectaron dos oportunidades que tienen su desarrollo dentro de un campus universitario que gestionan zonas verdes. La primera de ellas se refiere al uso del hormigón de desecho producto de las prácticas de laboratorio sobre materiales para la construcción, necesarias para la formación académica. Este material actualmente se desecha una vez las prácticas han terminado por lo que se desperdicia un material que aún conserva buenas propiedades mecánicas, que puede ser reutilizado completamente y que, como desecho, está contaminando al medio ambiente. Según los registros del laboratorio de docencia de materiales se desechan en promedio unas 6 toneladas de hormigón fresco al año. La segunda oportunidad es la existencia de plástico recuperado y clasificado en un punto limpio instalado dentro del Campus garantizando su disponibilidad permanente. Según ASIPLA (2019) la tasa de reciclaje de plástico en Chile a 2019 es de sólo un 8,5\% por lo que esta solución también promueve el uso de un material que hoy exige nuevos nichos de aplicación.

La incorporación de plástico del tipo PET como reemplazo de una porción de los agregados, en forma de fibras o como aditivo en una mezcla de hormigón ha sido estudiada en distintas investigaciones (Ciocan et al; 2018; Lakhiar et al., 2018; Poonyakan et al., 2018; Hameed y Ahmed, 2019). La fabricación de ladrillos de hormigón con adición de un $10 \%$ de PET permite obtener mezclas que cumplen con el valor mínimo requerido por la normativa local para las propiedades mecánicas de ladrillos según Infante-Alcalde y Valderrama-Ulloa (2019). Otros trabajos han investigado la posibilidad de fabricar hormigones livianos con la incorporación de distintas proporciones de plástico reciclado (Alqahtani et al., 2017; Al-Kaabi et al., 2020).

Por otra parte, la incorporación de fibras de PET en el hormigón se ha utilizado de manera efectiva para reducir las deformaciones ocasionadas por su retracción controlando la fisuración prematura y permitiendo alcanzar la ductilidad requerida (Koo et al., 2014; Bui et al., 2018). En cuanto a la resistencia a la compresión, las investigaciones sobre la adición de fibras PET al hormigón muestran resultados diferentes. Dinesh y Hanumantha Rao (2017) reportaron un aumento de la resistencia a la compresión medida en cubos con la incorporación de hasta un $0.5 \%$ de fibras PET dosificadas en volumen de la mezcla. Este aumento es de un $9.4 \%$ comparado con la muestra patrón. Un valor superior de incorporación de fibras PET arrojó una disminución de la resistencia a la compresión. Para un $1.0 \%$ de fibra PET se reportó una disminución de un $6 \%$ de la resistencia comparada con la mezcla con un $0.5 \%$ de fibras PET. Pelisser et al. (2012) desarrollaron una investigación incorporando fibras PET al hormigón en fracciones por volumen de $0 \%, 0.05 \%, 0.18 \%$ y $0.30 \%$. Se concluyó que la adición de fibras PET no influenciaron la resistencia a la compresión y el módulo de elasticidad del hormigón. En otro estudio experimental se encontró que la adición de fibra PET reciclada $0.5 \%, 0.75 \%$ y $1.0 \%$ dosificada como fracción del volumen de la mezcla exhibió una disminución de la resistencia a la compresión que osciló entre 1-9\% comparado con un hormigón sin refuerzo de fibras (Kim et al; 2010).

Otras investigaciones han demostrado que se incrementa la resistencia a la flexotracción comparado con un hormigón sin adición de fibras PET. Ramadevi y Manju (2012) concluyeron en un estudio que la resistencia a la flexión se incrementó hasta un $2 \%$ de reemplazo de los agregados finos por fibras PET en cerca de un $80 \%$ comparada con un hormigón sin adición de PET. Al-Hadithi et al. (2019) encontraron que el valor óptimo de la resistencia a la flexión se alcanzó con una adición de 1.5\% de fibra PET dosificada por volumen con un incremento del $70 \%$ comparado con la mezcla patrón (0\% PET). Por otro lado, Saikia y de Brito (2014) evaluaron la resistencia a la abrasión de hormigones con distintas tasas de reemplazo en volumen del agregado natural por PET triturado en dos fracciones (fina y gruesa). Este estudio concluyó que los mejores resultados fueron observados para un $10 \%$ de reemplazo de los agregados naturales para ambas fracciones de PET medidos en el ensayo de disco abrasivo de Böhme. Ali et al. (2020) encontraron que la inclusión de fibras PET al hormigón en reemplazo en volumen de los agregados tuvo un efecto positivo reduciendo la 
abrasión de la superficie con valores de pérdida de espesor del espécimen de ensayo de $1.1 \mathrm{~mm}$ para el $2 \%$ de fibra PET, lo que fue un $40 \%$ menor que el valor correspondiente para la mezcla control (sin fibras PET)

En relación con las ventajas medioambientales de construir elementos prefabricados de hormigón con adición de PET, se mostró la disminución de las emisiones de $\mathrm{CO}_{2}$ en la etapa de extracción de un 1,7\% para adiciones de 10\% de PET (Infante-Alcalde y Valderrama-Ulloa, 2019). En el trabajo de Sánchez et al. (2014) se indicó la disminución de un 10\% en la huella ecológica de un hormigón al incorporar un 20\% de PET, mientras que Usahanunth y Pochanart (2017) mostraron una variación en la disminución de emisiones de $\mathrm{CO}_{2}$ entre el $4,17 \%$ para el $1 \%$ de PET hasta un $31,60 \%$ con un $6 \%$ de PET en relación a un hormigón convencional.

La solución implica el diseño y fabricación de solerillas para la conformación de jardineras persiguiendo tres propósitos: 1) reutilizar dos materiales que actualmente son desechados y que requieren ser valorizados como el hormigón y el plástico PET en forma de fibras, 2) estructurar una propuesta de jardinera que sirva de recinto de almacenamiento de hojas caídas especialmente en las épocas del año donde este fenómeno aumenta y 3) facilitar la descomposición de las hojas para la producción de compost que sirva para el posterior abono de las zonas verdes y su vegetación. Y la adición de fibras de PET al hormigón para la fabricación de las solerillas tiene dos objetivos principales: 1) detener el crecimiento de microfisuras internas durante el proceso de curado permitiendo al hormigón alcanzar la ductilidad requerida y 2) permitir una manipulación y transporte de los elementos prefabricados más segura debido al mejoramiento en el desempeño de la resistencia a la flexotracción evitando su fractura o colapso repentino. La solución presentada promueve la innovación y avanza hacia un modelo más sustentable mediante el uso y gestión más eficiente de residuos, el fomento del reciclaje en los campus universitarios y parques públicos, y la reducción de la emisión de gases de efecto invernadero y del consumo energético. El uso de este tipo de jardineras puede ser adoptado por empresas o instituciones que presenten la misma problemática y que requieran de soluciones innovadoras y prácticas que incorporen los principios de la sustentabilidad en sus procesos de funcionamiento.

El principal objetivo de la investigación fue diseñar y fabricar elementos prefabricados de hormigón tipo solerilla para la confección de jardineras en amplias zonas verdes que puedan contener hojas muertas de los árboles, favoreciendo la actividad microbiana y optimizando las labores de mantenimiento. Para alcanzar este objetivo se desarrollaron las siguientes actividades: (1) diseño del prefabricado, (2) caracterización en laboratorio de las mezclas de hormigón utilizadas en las prácticas académicas de los cursos de materiales, (3) fabricación de los elementos prefabricados tipo solerilla y (4) análisis de los aspectos de sustentabilidad de la solución propuesta.

\section{OTROS ANTECEDENTES}

De acuerdo con el Código de Normas y Especificaciones Técnicas de Obras de Pavimentación del Ministerio de Vivienda y Urbanismo de Chile, una solerilla está definida como un elemento prefabricado de hormigón utilizado como límites de restricción para pavimentos de aceras, pasajes y sendas de circulación peatonal, así como también elementos de separación de áreas de jardines, plazas (MINVU, 2018). En Chile, estos prefabricados se clasifican según su forma y dimensiones en tipos A, B y C como se muestra en la Fig. 1 y son fabricadas en longitudes de $50 \mathrm{~cm}$ y $100 \mathrm{~cm}$.
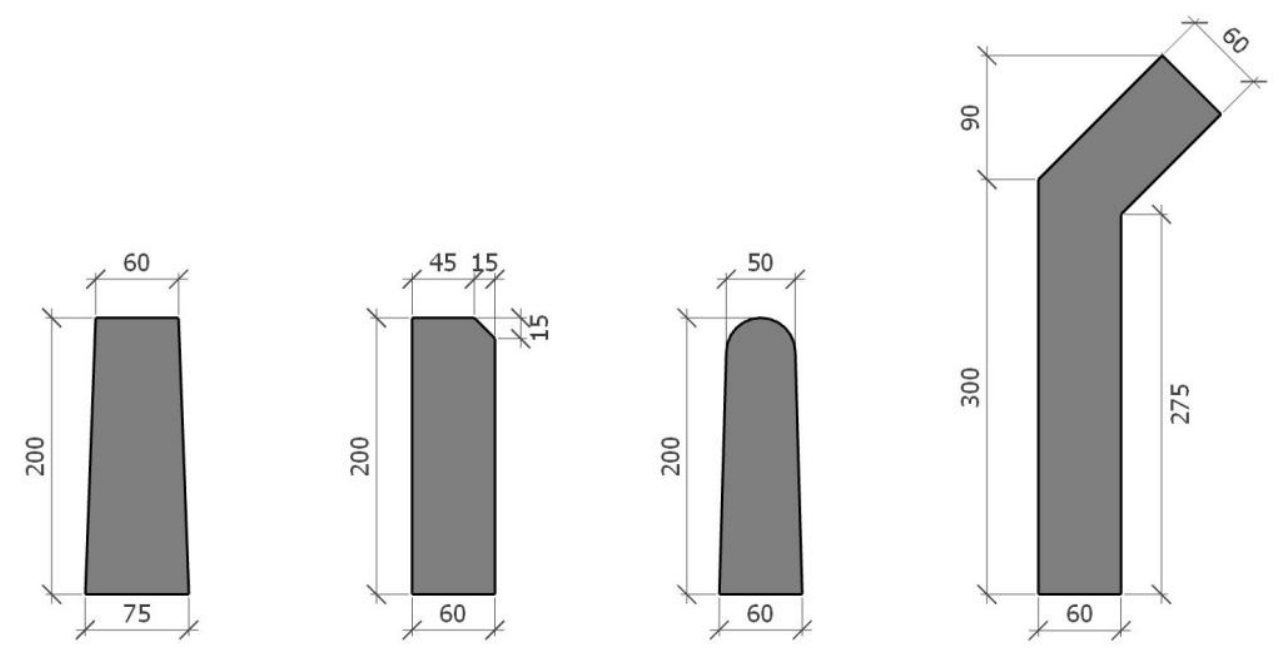

Fig. 1: Tipos de solerillas 
Esta investigación incluye el diseño de dos elementos prefabricados ensamblables que contempla una solerilla recta y una solerilla como módulo esquinero, con un diseño innovador como se muestra en la Fig 1 , solerilla propuesta, que permita un adecuado almacenamiento de la hojarasca. El diseño de las solerillas tiene como requerimientos: poder conformar una jardinera ensamblable de perímetro cerrado con un elemento modular prefabricado a base de hormigón y PET; asegurar un perímetro cerrado que sobresalga del nivel de suelo con la finalidad de contener un volumen de hojas secas en su interior y materializar un perímetro levemente reducido en la parte superior de la solerilla que impida el vaciado de hojas de la jardinera producto de movimiento de masas de aire. Tanto el moldaje como las solerillas deben ser de fácil fabricación puesto que serán fabricados por estudiantes como parte de su proceso de aprendizaje. Finalmente, las solerillas deben ser manuportables; es decir, su tamaño y peso deben permitir ser transportados por una persona.

Con los requerimientos de diseño establecidos se diseñó una solerilla recta de dimensiones $430 \mathrm{~mm}$ de longitud, $390 \mathrm{~mm}$ de altura y $60 \mathrm{~mm}$ de espesor. Además, se diseñó un elemento solerilla esquinera que permite conformar ángulos rectos con la finalidad de cerrar el perímetro de la jardinera. Las dimensiones en planta del elemento esquinero son $176 \mathrm{~mm}$ x $176 \mathrm{~mm}, 390 \mathrm{~mm}$ de altura y $60 \mathrm{~mm}$ de espesor (Fig. 2a). Tanto la solerilla recta como la solerilla esquinera tienen un sistema de ensamble macho y hembra en base a una lengüeta y una hendidura de iguales dimensiones lo que permite un encaje perfecto. Con un peso total para la solerilla recta de $24,21 \mathrm{~kg}$ y para la solerilla esquinera de $15,28 \mathrm{~kg}$, ambas cumplen con lo estipulado en la modificación de la Ley 20.949 conocida como "Ley del Saco" que limita a 25 kg el peso máximo de carga por una persona de sexo masculino, para evitar dolores de espalda, limitar el esfuerzo en los discos intervertebrales e incluso lesiones a extremidades superiores, de hombros (MTPS, 2016).

La disposición de ambos elementos, solerilla recta y solerilla esquinera, permite conformar diferentes tipos de jardinera. Así, cuatro módulos de solerilla y cuatro elementos esquineros permiten conformar una jardinera de planta cuadrada (Fig. 2b). Esa misma jardinera puede aumentar su capacidad agregando módulos de solerilla recta tanto en el ancho como en el largo (Fig. 2c). De esa manera puede responderse a diferentes configuraciones de tamaño según los requerimientos de capacidad de la jardinera y del espacio disponible para su instalación.

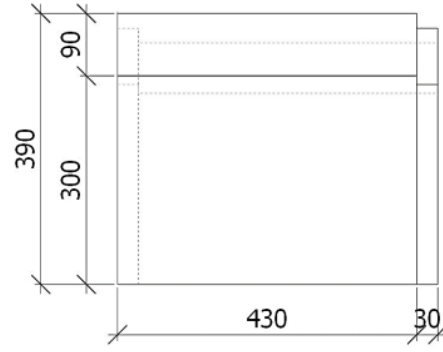

a)

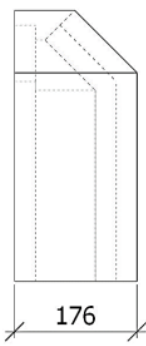

76

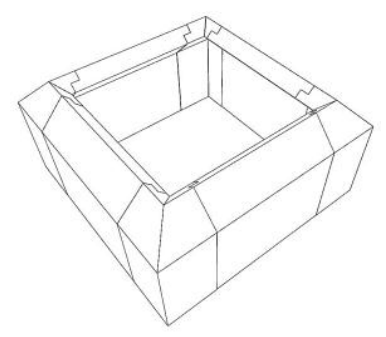

b)

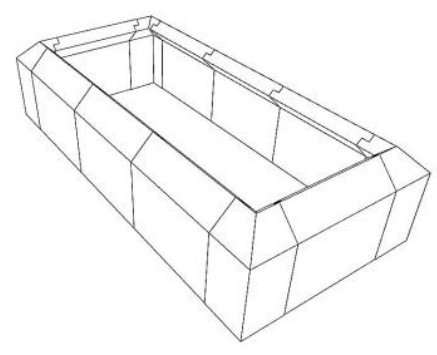

c)

Fig. 2: a) vista de solerilla recta y solerilla esquinera, b) configuración en planta cuadrada y c) configuración en planta rectangular

\section{MATERIALES Y MÉTODOS}

A continuación, se describen los materiales utilizados en esta investigación y los métodos experimentales de laboratorio aplicados a las mezclas de hormigón para la fabricación de las solerillas. El hormigón para la construcción de estos elementos prefabricados se considera de uso no estructural sólo destinado a conformar volúmenes de material resistente y requiere una resistencia característica a la compresión mínima de $15 \mathrm{MPa}$ a la compresión cilíndrica. Esta condición de baja resistencia hace posible la incorporación de materiales como el plástico reciclado.

\section{Materiales}

Los materiales utilizados en esta investigación fueron arena, gravilla, grava, cemento, agua y plástico reciclado. Los agregados gruesos y finos utilizados en esta investigación cumplieron los requisitos de la NCh163. El módulo de finura de la arena es de 3.06 que se encuentra en el rango de 2.90 y 3.20 por lo que clasifica como un agregado ligeramente grueso. El tamaño máximo de los agregados gruesos es de $11 / 2$ ". La proporción de mezcla de los agregados fue de $45 \%$ gruesos (grava y gravilla) y $55 \%$ finos (arena) y su gradación se muestra en la Fig. 3. La Tabla 1 presenta las propiedades físicas de los agregados. El cemento utilizado para todas las mezclas de investigación fue del tipo Portland comercial tipo A sin adición. Se utilizó agua potable para todas las mezclas elaboradas. 


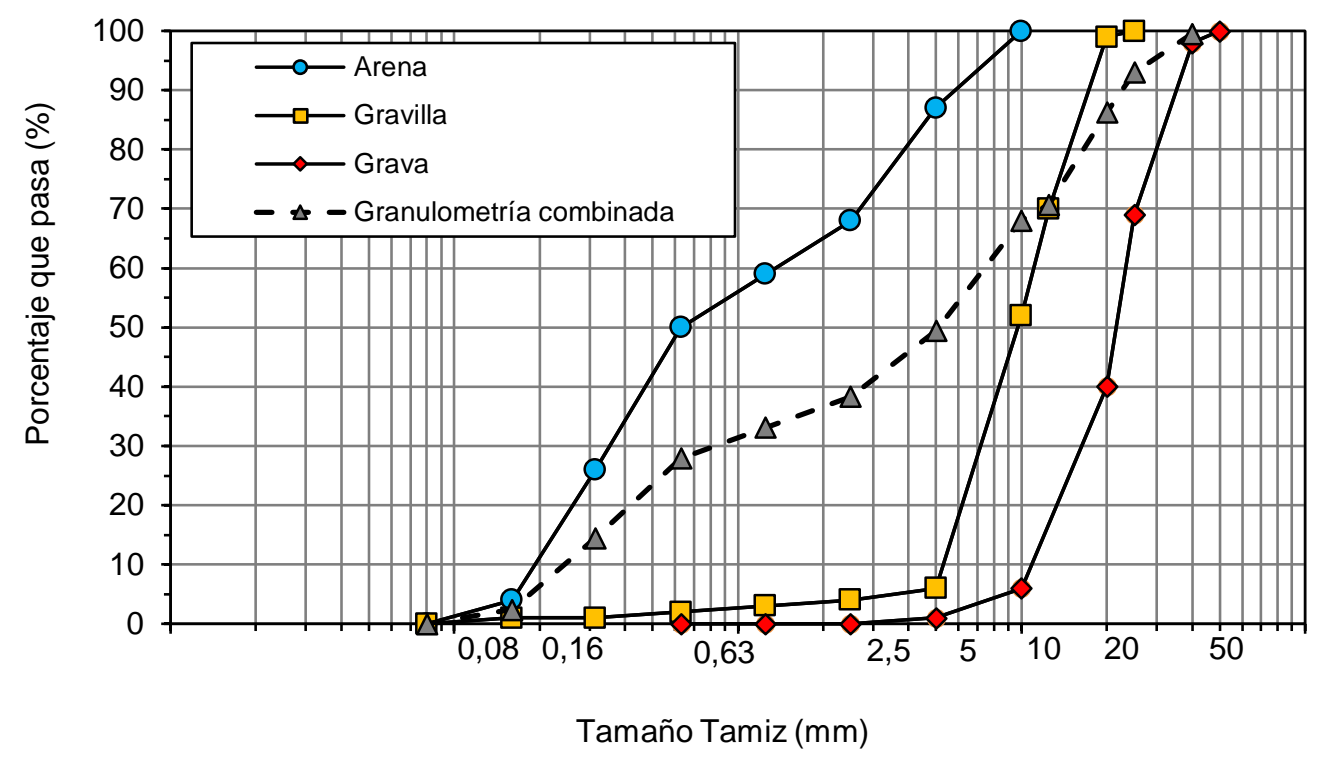

Fig. 3: Curvas de gradación para los agregados vírgenes finos y gruesos

Tabla 1: Propiedades físicas de los agregados

\begin{tabular}{|l|c|c|c|}
\hline \multirow{2}{*}{\multicolumn{1}{c|}{ Propiedad }} & \multicolumn{3}{c|}{ Tipo de Agregado } \\
\cline { 2 - 4 } & Arena & Gravilla & Grava \\
\hline Densidad real seca $\left(\mathrm{kg} / \mathrm{m}^{3}\right)$ & 2.620 & 2.630 & 2.650 \\
\hline Densidad real saturado superficialmente seco $\left(\mathrm{kg} / \mathrm{m}^{3}\right)$ & 2.660 & 2.670 & 2.670 \\
\hline Densidad neta $\left(\mathrm{kg} / \mathrm{m}^{3}\right)$ & 2.710 & 2.750 & 2.720 \\
\hline Absorción $(\%)$ & 1,25 & 1,60 & 1,10 \\
\hline Huecos $(\%)$ & 34,4 & 37,8 & 37,7 \\
\hline
\end{tabular}

El plástico reciclado corresponde a Polietileno de Tereftalato (PET) proveniente de botellas recuperadas para ser recicladas y fue adicionado en proporciones de $0 \%, 0,5 \%$ y $1,0 \%$ en peso total de la mezcla. Se utilizaron botellas plásticas de bebida de $250 \mathrm{~cm}^{3}$. A cada botella se le cortó la base y el cuello dejando el cuerpo como la parte reutilizable, de la que se obtuvo hasta 6 gramos de fibra (Fig. 4a). Del cuerpo de cada botella se extrajeron las fibras utilizando una herramienta manual de corte (Fig 4b). Se usaron fibras de PET de $5 \mathrm{~mm}$ de ancho y 50 $\mathrm{mm}$ de largo como se muestra en la Fig. 4c. La adición de estas fibras resulta conveniente para que los elementos prefabricados puedan manipularse y transportarse con un menor riesgo de rotura por flexión.

La cantidad de materiales utilizados para la fabricación de las solerillas se muestran en la Tabla 2. Si se toma como referencia la adición de $0,5 \%$ de PET, la cantidad de plástico requerido para una solerilla recta se consigue con el uso de 20 botellas de $250 \mathrm{~cm}^{3}$ y para una solerilla esquinera con 13 botellas y en el caso de la adición del $1 \%$ las cantidades serán las dobles. Y si se recupera todo el hormigón que es desechado en laboratorio de docencia (6 toneladas anuales), se podrían fabricar aproximadamente 392 solerillas rectas y con una adición de 0,5\% PET, se requerirían 7.840 botellas plásticas equivalentes a 47,8 kg de PET reutilizable anualmente y en el caso de la adición de $1 \%$ PET el doble de botellas.

\section{Procedimiento de mezclado y curado del hormigón}

Se utilizó una relación $\mathrm{A} / \mathrm{C}$ constante de 0,52 para todas las mezclas. Este valor permanece constante durante todas las prácticas de laboratorio en el año. Se dosificó según lo indicado por NCh 170:1985 para una resistencia característica a la compresión de cubos del hormigón de $20 \mathrm{MPa}$ (valor equivalente cercano a los $15 \mathrm{MPa}$ en términos de resistencia a la compresión en cilindros). Todos los agregados se sometieron a un proceso de saturación por 24 horas, previo para la fabricación de los hormigones y se realizó el ajuste respectivo del agua de mezcla según la propiedad de absorción. Para la fabricación de las mezclas se utilizó una betonera de 40 litros de capacidad. 


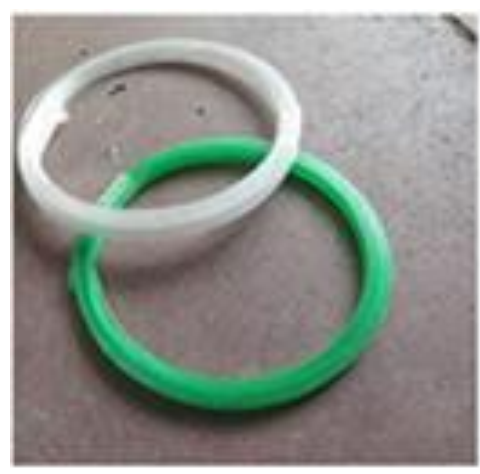

a

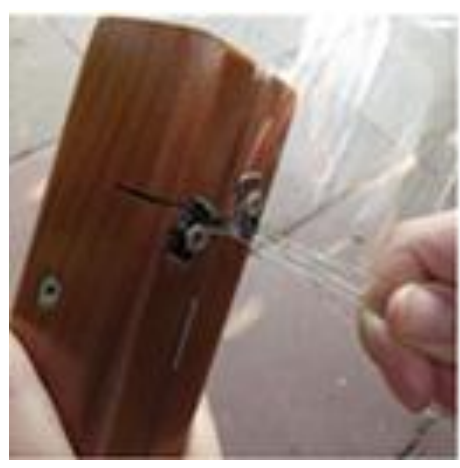

b

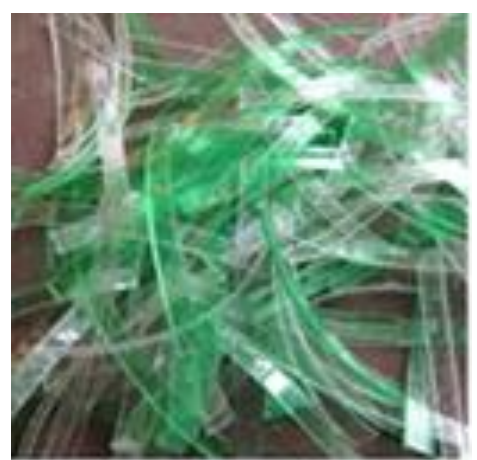

C

Fig. 4: Fibras PET utilizadas

Tabla 2: Cantidades de materiales para la fabricación por tipo de solerilla

\begin{tabular}{|l|c|c|c|}
\hline \multirow{2}{*}{ Tipo de solerilla } & \multirow{2}{*}{ Volumen de hormigón ocupado $\left(\mathrm{m}^{3}\right)$} & \multicolumn{2}{|c|}{ Cantidad de PET incorporado $(\mathrm{g})$} \\
\cline { 3 - 4 } & & $0,5 \%$ & $1,0 \%$ \\
\hline Recta & 0,01033 & 122 & 244 \\
\hline Esquinera & 0,00652 & 77 & 153 \\
\hline
\end{tabular}

El procedimiento de mezclado de los materiales es similar al utilizado por Valdés y Rapimán (2007) para la elaboración de ladrillos con materiales reciclados. Antes de la adición del agua, los agregados finos y gruesos, y el cemento fueron mezclados en condiciones secas hasta su homogeneización. Posteriormente se adicionó el agua y se realizó un proceso de mezclado por un tiempo aproximado de 3 minutos. Una vez las mezclas de hormigón fueron utilizadas en las prácticas de laboratorio, se procedió a adicionar las fibras PET dosificadas en función al peso total de la mezcla. Su homogeneización se logró mediante mezclado manual. Todas las mezclas fueron compactadas en una mesa vibratoria y curadas en piscina de inmersión a temperatura controlada de 20 ${ }^{\circ} \mathrm{C}$ por un período de 28 días. La Tabla 3 presenta las distintas proporciones de los materiales utilizados para la fabricación de las mezclas de hormigón.

Tabla 3. Proporciones de los materiales para las mezclas de hormigón

\begin{tabular}{|l|c|c|c|c|c|c|}
\hline \multirow{2}{*}{ Descripción } & \multicolumn{6}{|c|}{ Materiales $(\mathrm{kg})$} \\
\cline { 2 - 7 } & Agua & Cemento & Grava & Arena & PET 0,5\% & PET 1,0\% \\
\hline Por capacidad de betonera (40 litros) & 8,1 & 15,6 & 38,7 & 32,0 & 0,47 & 0,93 \\
\hline Por $\mathrm{m}^{3}$ de hormigón & 203 & 390 & 967 & 800 & 11,70 & 23,30 \\
\hline
\end{tabular}

\section{Métodos de ensayo}

Las mezclas de hormigón fueron ensayadas en dos etapas. La primera etapa consistió en el análisis de mezclas de hormigón fabricadas con agua a tres diferentes temperaturas $\left(15,20\right.$ y $\left.25^{\circ} \mathrm{C}\right)$ simulando distintas condiciones de mezclado. Este análisis tenía como objetivo evaluar la variación de algunas de las propiedades del hormigón debido a la temperatura del agua de mezclado. Estas mezclas se denominaron A, B y C cuando se empleó agua a una temperatura de 15,20 y $25{ }^{\circ} \mathrm{C}$ respectivamente. Se fabricaron 5 especímenes para cada mezcla de hormigón a las cuales se les determinó la densidad aparente en estado fresco aplicando la norma NCh 1564 y la resistencia a la compresión de probetas cúbicas de dimensiones $150 \mathrm{~mm}$ por $150 \mathrm{~mm}$ por $150 \mathrm{~mm}$ en estado endurecido mediante la norma NCh 1037. La segunda etapa, evaluó la resistencia a la compresión de hormigones fabricados con agua a las tres temperaturas de la etapa 1 con distintas adiciones de PET $(0,0,5$ y $1,0 \%)$ sobre el peso de los agregados, para lo cual se fabricaron tres especímenes cúbicos por mezcla. La norma empleada fue la NCh 1037. Todos los ensayos de los especímenes fueron realizados a los 28 días de curado. El objetivo de esta etapa fue evaluar en laboratorio el efecto de la incorporación de PET en la resistencia a la compresión de una mezcla de hormigón.

\section{RESULTADOS Y DISCUSIÓN}

Se presentan los resultados de la caracterización en laboratorio de las mezclas de prueba de hormigón sin y con la incorporación de PET tanto en su estado fresco como endurecido, se presentan los detalles de la fabricación del prefabricado tipo solerilla recta, las indicaciones para la posterior construcción de la jardinera y los principales aspectos relacionados con la sustentabilidad que se logran con la solución propuesta. 


\section{Propiedades del hormigón en estado fresco}

Los datos de densidad en estado fresco mostrados en la Tabla 4 fueron analizados mediante el criterio de ANOVA y Tukey para determinar si los resultados son significativamente distintos o no. El nivel de significancia o valor $p$ utilizado como referencia fue de 0.05 . Se obtuvieron valores de nivel de significancia mayores al valor de referencia ( $p$ valor: $A N O V A=0.122$; $p$ valores: mezclas $A B=0.587$, mezclas $A C=0.104$ y mezclas $B C=0.458$ ), por lo que se puede concluir que no existen diferencias significativas entre las densidades de las distintas mezclas de estudio. Por otro lado, se observa que los coeficientes de variación para las distintas mezclas muestran poca variabilidad de los resultados con valores inferiores al 10\%, indicando que los datos son homogéneos y consistentes.

Tabla 4. Densidad del hormigón en estado fresco

\begin{tabular}{|c|c|c|c|c|c|c|}
\hline \multirow{2}{*}{ Mezcla } & \multirow{2}{*}{ Muestra } & \multicolumn{2}{|c|}{ Densidad $\left(\mathrm{kg} / \mathrm{m}^{3}\right)$} & \multirow{2}{*}{$\begin{array}{l}\text { Desviación } \\
\text { estándar }\end{array}$} & \multirow{2}{*}{$\begin{array}{c}\text { Error estándar } \\
\text { (95\% nivel de confianza) }\end{array}$} & \multirow{2}{*}{$\operatorname{cov}(\%)$} \\
\hline & & Muestra & Promedio & & & \\
\hline \multirow{5}{*}{ A } & 1 & 2.339 & \multirow{5}{*}{2.316} & \multirow{5}{*}{17,88} & \multirow{5}{*}{15,68} & \multirow{5}{*}{0,77} \\
\hline & 2 & 2.323 & & & & \\
\hline & 3 & 2.290 & & & & \\
\hline & 4 & 2.315 & & & & \\
\hline & 5 & 2.311 & & & & \\
\hline \multirow{5}{*}{ B } & 1 & 2.390 & \multirow{5}{*}{2.333} & \multirow{5}{*}{35,26} & \multirow{5}{*}{30,91} & \multirow{5}{*}{1,51} \\
\hline & 2 & 2.319 & & & & \\
\hline & 3 & 2.299 & & & & \\
\hline & 4 & 2.315 & & & & \\
\hline & 5 & 2.340 & & & & \\
\hline \multirow{5}{*}{ C } & 1 & 2.316 & \multirow{5}{*}{2.353} & \multirow{5}{*}{24,05} & \multirow{5}{*}{21,08} & \multirow{5}{*}{1,02} \\
\hline & 2 & 2.365 & & & & \\
\hline & 3 & 2.377 & & & & \\
\hline & 4 & 2.344 & & & & \\
\hline & 5 & 2.365 & & & & \\
\hline \multicolumn{3}{|c|}{ Promedios } & 2.334 & 29.46 & 14,91 & 1,26 \\
\hline
\end{tabular}

\section{Propiedades del hormigón en estado endurecido}

La resistencia a la compresión fue la propiedad medida en laboratorio para los hormigones de prueba. Esta propiedad se obtuvo del ensayo de especímenes cúbicos. Para estimar la resistencia a la compresión cilíndrica a partir de los valores de resistencia a la compresión cúbica se utilizó un factor empírico de 0,8 comprendido en el rango recomendado en la literatura que oscila entre 0,78 y 0,89 (Pacheco et al., 2019; Srinivasa Reddy et al., 2019; Gyurkó y Nemes, 2020).

La Fig. 5 presenta los resultados de la resistencia a la compresión cúbica de los distintos hormigones fabricados con agua a distintas temperaturas para la primera etapa de la investigación. Se observa que todas las resistencias son similares, lo que se esperaba debido a que a la edad de ensayo de 28 días el hormigón prácticamente obtiene su máxima resistencia independientemente de que las mezclas se hayan elaborado con agua a distintas temperaturas. Se obtuvieron coeficientes de variación menores al $10 \%$ lo que indica que los datos son homogéneos y consistentes. Las barras representan el error estándar con un $95 \%$ de confiabilidad. Estos valores corresponden a una resistencia cilíndrica de 17,8, 19,0, 17,4 y 18,0 MPa para las mezclas A, B, C y promedio respectivamente utilizando el factor empírico recomendado. Los datos indican que es factible obtener hormigones con resistencia a la compresión cilíndrica superior a los $15 \mathrm{MPa}$ requeridos y que pueden ser empleados en la fabricación de elementos prefabricados como soleras o solerillas para uso no estructural. También se observa que las resistencias a la compresión son similares independientemente de la temperatura del agua de mezclado por lo que es factible utilizar cualquiera de las temperaturas estudiadas.

Los resultados de la resistencia a la compresión cúbica para las mezclas de hormigón con adición de fibras PET para la segunda etapa se muestran en la Fig. 6. Las barras de error representan el error estándar para una confiabilidad del $95 \%$. Se observa que los valores promedio de las resistencias a la compresión de las mezclas con adición de PET son mayores comparado con la mezcla patrón. Existe un aumento del $13.5 \%$ en la resistencia para las mezclas con adición de $0.5 \%$ de PET comparado con las mezclas patrón. Al incorporar un $1.0 \%$ de PET se obtuvo una disminución de la resistencia a la compresión de un $7.9 \%$ comparada con las mezclas con $0.5 \%$ de PET, lo que podría deberse al aumento de las zonas de transición interfacial (ITZ) entre las fibras de plástico y el mortero del hormigón. 


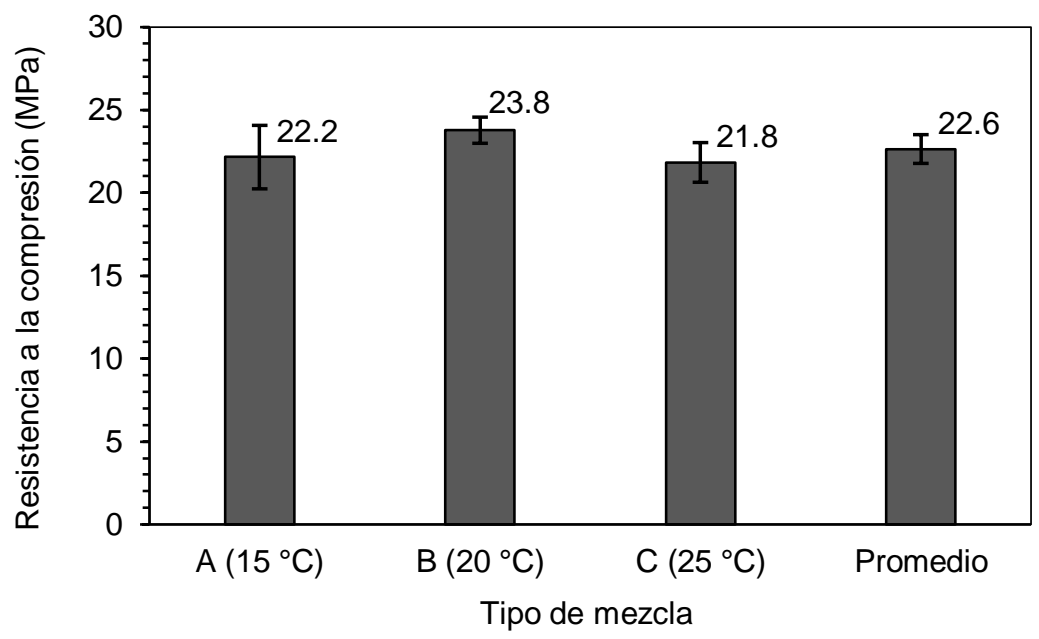

Fig. 5: Resistencia a la compresión cúbica de hormigones con adición de agua a distintas temperaturas - Etapa 1

Con una mayor cantidad de fibras en la mezcla, su distribución se hace más irregular por lo que la existe una menor trabajabilidad, una consolidación incompleta y la adhesión entre la superficie de la fibra y la pasta de cemento se altera produciendo zonas ITZ más débiles influyendo en las propiedad de resistencia a la compresión del hormigón (Afroughsabet et al., 2016). Esta misma tendencia fue reportada por Dinesh y Hanumantha Rao (2017), indicando que existió un incremento de la resistencia a la compresión medida en cubos de un $9.4 \%$ aproximadamente cuando se incorporó el $0.5 \%$ de fibras PET medido en volumen de la mezcla comparado con la mezcla patrón sin adición de fibras. También se observó una disminución en un $5.5 \%$ de la resistencia a la compresión cuando se incorporó un 1.0\% PET comparado con el 0.5\% PET en las mezclas.

Lo anterior indica que es factible incorporar PET en las mezclas de hormigón y obtener un desempeño en cuanto a su resistencia a la compresión superior a las mezclas sin adición de PET. Los valores de la resistencia a la compresión cilíndrica equivalente utilizando el factor empírico recomendado son 16.0, 18.2 y $16.7 \mathrm{MPa}$ para las mezclas patrón, con adiciones de $0.5 \%$ y $1.0 \%$ PET respectivamente. Estos valores son superiores al mínimo exigido de $15 \mathrm{MPa}$ para la fabricación de las solerillas. Los autores sugieren la incorporación del $0.5 \%$ debido a que se obtiene la resistencia mínima requerida para la fabricación del elemento prefabricado. Se deben hacer otros estudios para determinar la posibilidad de incorporar porcentajes superiores al $1.0 \%$ estimando el efecto que produce sobre las propiedades mecánicas del hormigón.

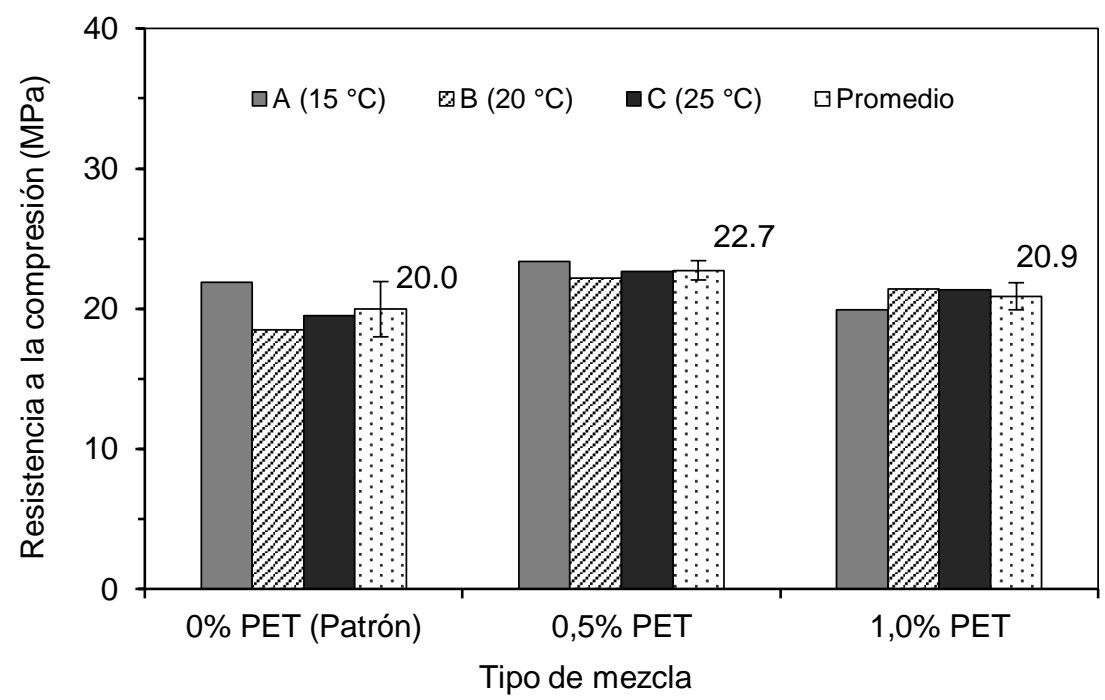

Fig. 6: Resistencia a la compresión cúbica de hormigones con adición de agua a distintas temperaturas y distintos contenidos de PET- Etapa 2 


\section{Fabricación del prefabricado}

El proceso de fabricación contempla la obtención de los dos tipos de solerillas. Para el armado del moldaje se utilizó una placa de madera contrachapada de espesor $15 \mathrm{~mm}$. El moldaje, que permite la fabricación de una solerilla a la vez, se compone de cinco piezas de contrachapado atornillado y dos tapas laterales (Fig. 7). Respecto a su durabilidad, cada moldaje está diseñado para cuatro usos antes de su reposición. Para evitar que el molde se adhiera a la superficie de la solerilla se aplicó un agente desmoldante de emulsión líquida diseñado especialmente para encofrados de madera permitiendo así prolongar la vida útil del moldaje.

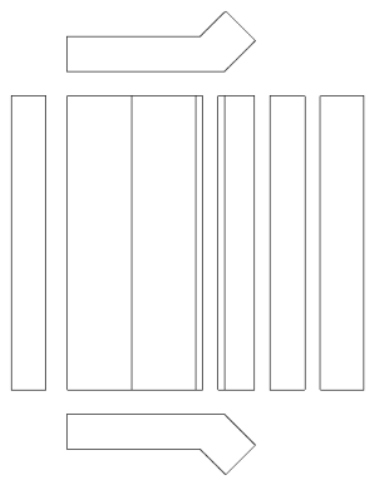

a)

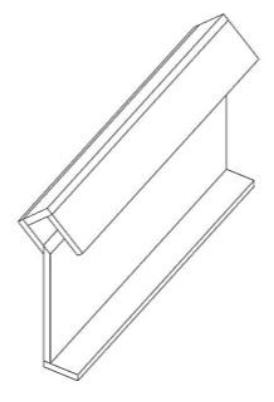

b)

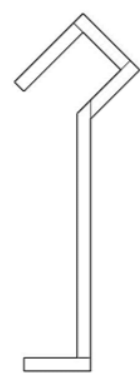

c)

Fig. 7: Despiece a), isométrica b) y corte del moldaje c)

Para la fabricación de la solerilla recta se dispuso el moldaje directamente sobre una mesa vibratoria con la finalidad de compactar la mezcla durante el proceso de hormigonado y evitar así posibles defectos por mala distribución de la mezcla (Fig 8a). Posteriormente se aplicó un proceso de curado por tres días cubriendo la solerilla con polietileno para evitar la pérdida de humedad y permitir el correcto fraguado del hormigón (Fig 8b). Finalmente, la solerilla fue sometida a un proceso de inmersión en agua hasta completar los 28 días de curado total y fue acopiada en el laboratorio (Fig 8c).

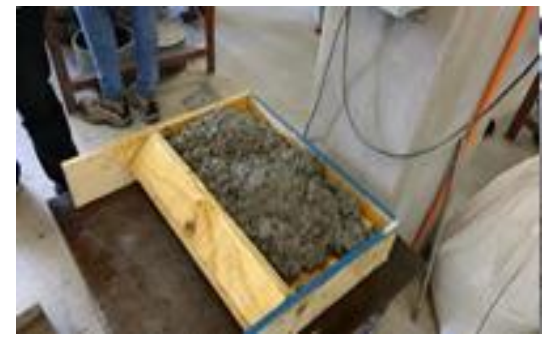

a)

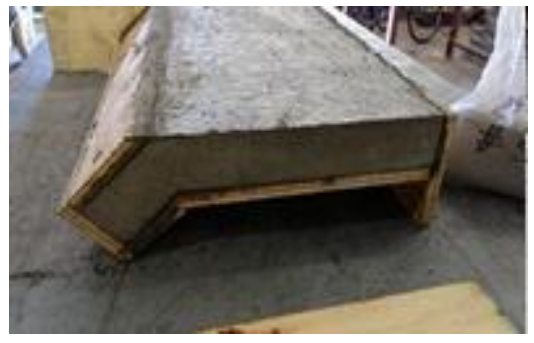

b)

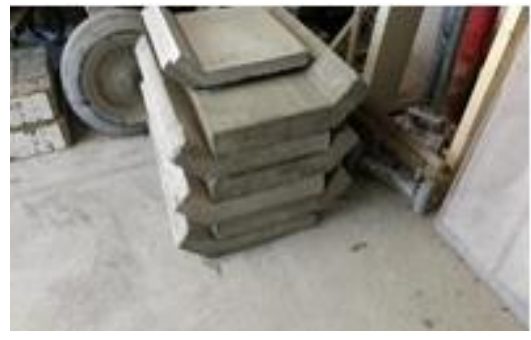

c)

Fig. 8: Proceso de fabricación de la solerilla

\section{Construcción de la jardinera}

El montaje de las solerillas para la materialización de la jardinera debe cumplir lo estipulado en el Artículo 6.6 de MINVU (2018). La secuencia de montaje de la solerilla recta y la solerilla esquinera considera tres etapas bien marcadas: (1) excavación del terreno (Fig. 9a), (2) montaje de solerilla recta y solerilla esquinera (Fig. 9b) y (3) cierre del perímetro de la jardinera con los módulos solerilla recta y solerilla esquinera (Fig. 9c).

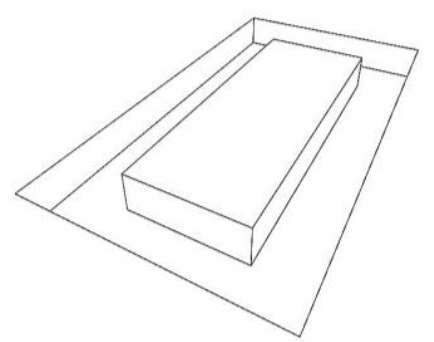

a)

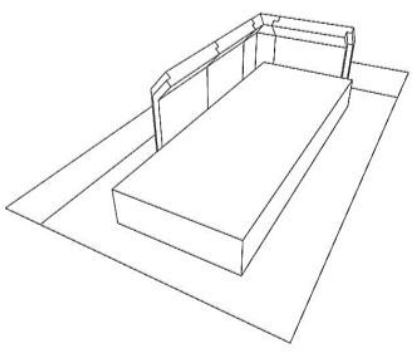

b)

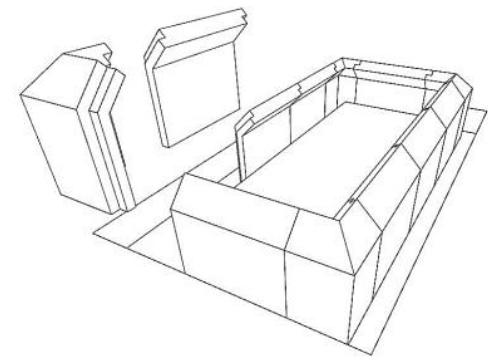

c)

Fig. 9: Secuencia de montaje de la jardinera 


\section{Aspectos de sustentabilidad logrados}

Además de los beneficios logrados por la solución de jardineras construidas con solerillas para el almacenamiento de las hojas caídas de los árboles y la optimización en el tiempo de trabajo para su recolección, se destacan los aspectos de reutilización de un hormigón actualmente desechado y plástico reciclado pendiente de su valorización a través de la fabricación de una solerilla para jardinera. Los aspectos de sustentabilidad logrados considerados más importantes se relacionan con la cantidad de material reutilizado, la reducción de las emisiones de $\mathrm{CO}_{2}$ emitidas al medio ambiente y la disminución del consumo energético. Para lograr esto se utilizó la metodología de cálculo de huella de carbono explicada en el trabajo de Infante-Alcalde y Valderrama-Ulloa (2019), los factores de emisión de $\mathrm{kgCO}_{2} / \mathrm{t}$ de Miranda (2020) y de Athena Sustainable Materials Institute, (1998). Los factores de emisión para las energías utilizadas en la extracción de materiales fueron de 69,3 ( $\left.\mathrm{kgCO}_{2} / \mathrm{TJ}\right)$ para gasolina, petróleo combustible 77,4 ( $\left.\mathrm{kgCO}_{2} / \mathrm{TJ}\right)$ y electricidad 0,4147 tCO 2 eq/MWh (ME, 2020).

En cuanto a la reutilización de materiales se destaca el uso de 3.250 litros de hormigón fresco utilizado anualmente para el desarrollo de las prácticas de laboratorio de materiales en la Escuela de Construcción Civil equivalente a una masa aproximada de 6 toneladas o 2,6 $\mathrm{m}^{3}$. Con esta propuesta de prefabricados es posible disminuir las emisiones de $\mathrm{CO}_{2}$ eq y el consumo energético al eliminar la etapa de extracción de materias primas, ya que es la etapa que se evita puesto que se reutilizan las mismas materias primas para fabricar las solerillas propuestas, como se muestra en la Tabla 5. La disminución por cada solerilla recta es de 0,16 $\mathrm{kgCO}_{2} \mathrm{eq}$ y de consumo energético de 5,42 kWh, por lo que si la totalidad del hormigón fresco se utiliza en la fabricación de 248 solerillas rectas con un $1 \%$ de PET es posible evitar anualmente la emisión de $40,66 \mathrm{kgCO}_{2}$ eq y disminuir el consumo energético en $1.345,40 \mathrm{kWh}$.

Tabla 5: Emisión de CO2eq y consumo energético para $1 \mathrm{~m}^{3}$ de hormigón, para el producto solerilla y para todo el hormigón reutilizado

\begin{tabular}{|c|c|c|c|c|}
\hline \multicolumn{2}{|c|}{ Producto Elaborados } & \multirow{2}{*}{$\frac{\mathrm{kgCO}_{2 \mathrm{eq}}}{0,30}$} & \multirow{2}{*}{$\begin{array}{c}\mathrm{kWh} / \mathrm{m}^{3} \\
7,65\end{array}$} & \multirow{2}{*}{$\frac{F E\left(k g C O_{2} e q / t\right)}{2,9}$} \\
\hline \multirow{5}{*}{$\begin{array}{l}\text { Extracción Materias Primas } \\
\qquad\left(1 \mathrm{~m}^{3} \text { de hormigón) }\right.\end{array}$} & Agregado Grueso & & & \\
\hline & Agregado Fino & 0,67 & 24,58 & 5 \\
\hline & Cemento & 14,64 & 484,58 & 571 \\
\hline & PET (0,5\%) & 0,02 & 0,33 & 0,234 \\
\hline & PET (1\%) & 0,03 & 0,65 & 0,234 \\
\hline \multicolumn{2}{|l|}{$1 \mathrm{~m}^{3}$ de hormigón $0 \%$ PET } & 15,61 & 516,81 & \\
\hline \multicolumn{2}{|l|}{$1 \mathrm{~m}^{3}$ de hormigón 0,5\% PET } & 15,63 & 517,14 & \\
\hline \multicolumn{2}{|c|}{$1 \mathrm{~m}^{3}$ de hormigón 1,0\% PET } & 15,64 & 517,46 & \\
\hline \multicolumn{2}{|c|}{$\begin{array}{l}\text { Solerilla recta de } 430 \mathrm{~mm} \text { de longitud, } 390 \mathrm{~mm} \text { de altura y } 60 \\
\mathrm{~mm} \text { de espesor }\left(24,21 \mathrm{~kg}-0,01033 \mathrm{~m}^{3}\right)\end{array}$} & 0,16 & 5,42 & \\
\hline \multicolumn{2}{|c|}{$\begin{array}{l}\text { Solerilla esquinera de } 176 \mathrm{~mm} \times 176 \mathrm{~mm}, 390 \mathrm{~mm} \text { de altura } \\
\text { y } 60 \mathrm{~mm} \text { de espesor }\left(15,28 \mathrm{~kg}-0,00652 \mathrm{~m}^{3}\right)\end{array}$} & 0,10 & 3,40 & \\
\hline \multicolumn{2}{|c|}{ Utilización de 2,6 m³ de hormigón y 60,6 kg de PET } & 40,66 & $1.345,40$ & \\
\hline
\end{tabular}

Ambientalmente la solerilla demuestra ser un prefabricado más sustentable que otras solerillas ya que al fabricarse con hormigón de desecho y al reutilizarse PET se evitan emisiones de $\mathrm{CO}_{2}$ eq y consumo energético al eliminar la etapa de extracción de nuevas materias primas. Si se considera que los agregados ocupan el mayor volumen en la elaboración de la solerilla, su carga ambiental y energética es de sólo un $6 \%$ comparado con el $93 \%$ del cemento, por lo que los esfuerzos deben enfocarse en la utilización de cementos adicionados o más sustentables que permitan disminuir el impacto ambiental en todo su ciclo de vida.

Por otra parte, en la Tabla 6 se muestran los costos por $\mathrm{m}^{3}$ (en pesos chilenos - CLP) de la fabricación de cada una de las mezclas estudiadas, en paralelo se entrega el costo unitario de fabricación de la solerilla recta y luego el comparativo de precio unitario de venta (el que incorpora moldes, betonera, mano de obra y utilidades) que ha sido comparado con el precio unitario de una solerilla existente en el mercado con dimensiones comerciales similares (1000 mm longitud x $200 \mathrm{~mm}$ de altura $\times 60$ de espesor $\mathrm{mm}$ ) a la solerilla diseñada en la presente investigación. También se observa un aumento progresivo del costo unitario y precio unitario debido al aumento de costo por la adición de PET a la mezcla, ya que ese valor es el precio de compra PET a una empresa recicladora que se podría disminuir o eliminar si los estudiantes realizan el reciclado del PET en las instalaciones de laboratorio de docencia. Aún, así al comparar el precio unitario de venta de las solerillas con distinto porcentaje de adición PET, el precio sigue siendo competitivo un $9 \%$ más económico para la mezcla con $0,5 \%$ de PET y sólo un $9 \%$ más caro con la mezcla del $1 \%$ de PET. 
Tabla 6: Costo unitario de fabricación, precio unitario de venta de la solerilla propuesta y comparativo con una solerilla existente en el mercado

\begin{tabular}{|l|r|r|r|r|}
\hline \multicolumn{1}{|c|}{ Materia Prima } & Precio Unitario $(\$ C L P)$ & \multicolumn{1}{c|}{ Patrón } & \multicolumn{1}{c|}{ Mezcla 0,5\% PET } & Mezcla 1,0\% PET \\
\hline PET $(\mathrm{kg})$ & 200 & 0 & 2.340 & 4.660 \\
\hline Cemento $(\mathrm{kg})$ & 139,2 & 54.288 & 54.288 & 54.288 \\
\hline Arena $(\mathrm{kg})$ & 37,6 & 30.080 & 30.080 & 30.080 \\
\hline Grava $(\mathrm{kg})$ & 37,6 & 36.359 & 36.359 & 36.359 \\
\hline Total $(\$ / \mathrm{m} 3)$ & & 120.727 & 123.067 & 1.289 \\
\hline Costo unitario de materiales - solerilla recta (\$ CLP) & 1.264 & 1.448 & 1.313 \\
\hline Precio unitario de venta - solerilla recta (\$ CLP) & 1.421 & & 1.475 \\
\hline \multicolumn{2}{|l|}{ Precio unitario de venta - solerilla comercial (\$ CLP) } & & & 1.462 \\
\hline
\end{tabular}

\section{CONCLUSIONES}

De acuerdo con los resultados obtenidos y el análisis presentado se plantean las siguientes conclusiones principales: 1) la solución de elementos prefabricados tipo solerilla para la construcción de jardineras es una alternativa para reducir residuos como los hormigones en estado fresco que se desechan y la utilización de plástico para reciclado del tipo PET. Los autores sugieren una adición del $0.5 \%$ PET debido a que se obtiene la resistencia mínima requerida a la compresión y es un hormigón más económico comparado con las otras mezclas analizadas. Esta solución puede ser aplicada por empresas que gestionan amplias zonas verdes o por los parques públicos; 2) las jardineras pueden ser ajustables en tamaño obteniendo un área de almacenamiento acorde con las necesidades; 3) el hormigón desechado de las prácticas académicas universitarias cumplen con el requerimiento mínimo de resistencia para la fabricación de elementos prefabricados tipo solerilla con un uso no estructural; 4) existe una reducción de las emisiones de $\mathrm{CO}_{2}$ a la atmósfera y del consumo energético principalmente en la etapa de extracción de materias primas debido a la reutilización de materiales que actualmente son un desecho como el hormigón de las prácticas académicas y el plástico recuperado en botellas y 5) el diseño de las solerillas recta y esquinera cumple con lo estipulado por la Ley del Saco (modificación de Ley 20.949 del 17 de septiembre de 2016) vigente en Chile que limita a $25 \mathrm{~kg}$ el peso máximo que puede cargar una persona de sexo masculino.

\section{REFERENCIAS}

Athena Sustainable Materials Institute, Life cycle analysis of bricks and mortar products, 1a Ed., 1-114, George J. Venta P. Eng, Otawa, Canada (1998)

Afroughsabet, V., Biolzi, L., y Ozbakkaloglu, T. High-performance fiber-reinforced concrete: a review https://doi.org/10.1007/s10853-016-9917-4, Journal of Materials Science; 51 (14) (2016).

Al-Hadithi, A. I., Noaman, A. T. y Mosleh, W. K., Mechanical Properties and Impact Behavior of PET Fiber Reinforced Selfcompacting Concrete (SCC), https://doi.org/10.1016/j.compstruct.2019.111021, Composite Structures; 224, 1-12 (2019).

Ali, T. K. M., Hilal, N., Faraj, R. H. y Al-Hadithi, A. I., Properties of Eco-friendly Pervious Concrete Containing Polystyrene Aggregates Reinforced with Waste PET Fibers, https://doi.org/10.1007/s41062-020-00323-w, Innovative Infrastructure Solutions, 5(3) (2020).

Al-Kaabi, J., Al-Soudani, M. y Sharba, A., Practical Study on the Effect of Partial Replacement of Coarse Aggregate with Plastic Waste on Some Normal Concrete Properties, https://doi.org/10.1088/1757-899X/870/1/012036, IOP Conference Series: Materials Science and Engineering, 870(1), 012036 (2020).

Alqahtani, F. K., Khan, M. I., Ghataora, G., y Dirar, S., Production of Recycled Plastic Aggregates and Its Utilization in Concrete, https://doi.org/10.1061/(ASCE)MT.1943-5533.0001765, Journal of materials in civil engineering, 29(4) (2017).

ASIPLA - Asociación Gremial de Industriales del Plástico, Estudio sobre reciclaje de Plásticos en Chile, http://www.asipla.cl/, 1-13 (2019)

Bui, N. K., Satomi, T. y Takahashi, H., Recycling Woven Plastic Sack Waste and PET Bottle Waste as Fiber in Recycled Aggregate Concrete: An experimental study, https://doi.org/10.1016/j.wasman.2018.05.035, Waste Management, 78, 7993 (2018).

Ciocan, V., Burlacu, y otros 4 autores, Eco-friendly Concrete from Wastes, http://www.eemj.eu, Environmental engineering and management journal, 17(12), 2969-2976 (2018).

Dinesh, Y., \& Hanumantha Rao, C., Strength Characteristics of Fibre Reinforced Concrete Using Recycled PET, http://www.iaeme.com/, International Journal of Civil Engineering and Technology, 8(4), $92-99$ (2017).

Gyurkó, Z., y Nemes, R., Specimen Size and Shape Effect on the Compressive Strength of Normal Strength Concrete, https://doi.org/10.3311/PPci.15338, Periodica Polytechnica Civil Engineering, 64(1), 276-286 (2020).

Hameed, A. M., y Ahmed, B. A. F. Employment the Plastic Waste to Produce the Light Weight Concrete. https://doi.org/10.1016/j.egypro.2018.11.160, Energy Procedia, 157, 30-38 (2019). 
Infante-Alcalde, J. y Valderrama-Ulloa, C., Análisis Técnico, Económico y Medioambiental de la Fabricación de Bloques de Hormigón con Polietileno Tereftalato Reciclado (PET), https://doi.org/10.4067/S0718-07642019000500025, Información tecnológica, 30(5), 25-36 (2019)

Kim, S. B., Yi, N. H., Kim, H. Y., Kim, J. H. J., y Song, Y. C, Material and Structural Performance Evaluation of Recycled PET Fiber Reinforced Concrete, https://doi.org/10.1016/j.cemconcomp.2009.11.002, Cement and Concrete Composites, 32(3), 232-240 (2010).

Koo, B. M., Kim, J. H. J., Kim, S. B., y Mun, S., Material and Structural Performance Evaluations of Hwangtoh Admixtures and Recycled PET Fiber-added Eco-friendly Concrete for $\mathrm{CO}_{2}$ Emission Reduction, https://doi.org/10.3390/ma7085959, Materials, 7(8), 5959-5981 (2014).

Lakhiar, M. T., Sohu, S. y otros 4 autores, Flexural Performance of Concrete Reinforced by Plastic Fibers, https://etasr.com/index.php/ETASR/article/view/2084, Engineering technology \& applied science research, 8(3), 30413043 (2018).

ME, Ministerio de Energía - Gobierno de Chile, Factores de emisión, http://energiaabierta.cl/visualizaciones/factor-deemision-sic-sing/ (2020)

MINVU, Ministerio de Vivienda y Urbanismo - Gobierno de Chile, Código de Normas y Especificaciones Técnicas de Obras de Pavimentación, 141-172, Santiago, Chile (2018).

MTPS, Ministerio del Trabajo y previsión Social - Gobierno de Chile, LEY 20.949: Modifica modifica el código del trabajo para reducir el peso de las cargas de manipulación manual, http://bcn.cl/2f8n8, Biblioteca del Congreso Nacional (2016)

Miranda I. Propuesta de una metodología para estimar las emisiones de gases de efecto invernadero (GEl) y el consumo energético de un pavimento durante todo su ciclo de vida. Tesis de Magíster en construcción, Pontificia Universidad Católica de Chile (2020).

NCH163: Inst. Nacional de Normalización (Chile), Áridos para morteros y hormigones - Requisitos, Santiago-Chile (2013) NCH170: Inst. Nacional de Normalización (Chile), Hormigón - Requisitos generales, Santiago-Chile (1985)

NCH1037: Inst. Nacional de Normalización (Chile), Hormigón - Ensayo de compresión de probetas cúbicas y cilíndricas, Santiago-Chile (2009)

NCH1564: Inst. Nacional de Normalización (Chile), Hormigón - Determinación de la densidad aparente, del rendimiento, del contenido de cemento y del contenido de aire del hormigón fresco, Santiago-Chile (2009)

Pacheco, J. N., de Brito, J., Chastre, C., y Evangelista, L., Probabilistic Conversion of the Compressive Strength of Cubes to Cylinders of Natural and Recycled Aggregate Concrete Specimens, https://doi.org/10.3390/ma12020280, Materials, 12(2) (2019).

Pelisser, F., Montedo, O., Gleize, P. y Roman, H., Mechanical Properties of Recycled PET Fibers in Concrete, https://doi.org/10.1590/S1516-14392012005000088, Materials Research, 15(4), 679-686 (2012).

Poonyakan, A., Rachakornkij, M., Wecharatana, M., y Smittakorn, W., Potential Use of Plastic Wastes for Low Thermal Conductivity Concrete, https://doi.org/10.3390/ma11101938, Materials, 11(10) (2018).

Ramadevi, K. y Manju, R., Experimental Investigation on the Properties of Concrete with Plastic PET (bottle) Fibres as Fine Aggregates. International journal of emerging technology and advanced engineering, 2(6), 42-46 (2012)

Saikia, N. y de Brito, J., Mechanical Properties and Abrasion Behaviour of Concrete Containing Shredded PET Bottle Waste as a Partial Substitution of Natural Aggregate, https://doi.org/10.1016/j.conbuildmat.2013.11.049, Construction and building materials, 52, 236-244 (2014).

Sánchez, I., Oshiro, A. y Positieri, M., The Use of Recycled Plastic in Concrete. An Alternative to Reduce the Ecological Footprint. Journal of Construction, 13(3), 19-26 (2014).

Srinivasa Reddy, V., Seshagiri Rao, M. V. y Shrihari, S., Strength Conversion Factors for Concrete Based on Specimen Geometry, Aggregate Size and Direction of Loading. https://doi.org/10.35940/ijrte.B2336.078219, International Journal of Recent Technology and Engineering, 8(2), 2125-2130 (2019).

Usahanunth, N., y Pochanart, P., CO2-emission Assessment of the Concrete Added Crushed PET Bottles Waste, https://doi.org/10.14456/jtir.2017.2, Journal of Thai Interdisciplinary Research, 12(1), 9-15 (2017).

Valdés, G. A., y Rapimán, J. G. Propiedades físicas y mecánicas de bloques de hormigón compuestos con áridos reciclados. https://doi.org/10.4067/S0718-07642007000300010, Información tecnológica, 18(3), 81-88 (2007). 